\title{
Beyond Stroke Prevention in Atrial Fibrillation: Exploring Further Unmet Needs with Rivaroxaban
}

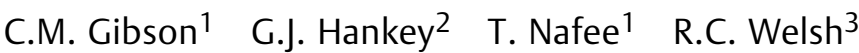 \\ ${ }^{1}$ Division of Cardiovascular Medicine, Beth Israel Deaconess Medical \\ Center, Harvard Medical School, Boston, Massachusetts, \\ United States \\ 2 Medical School, Faculty of Health and Medical Sciences, The \\ University of Western Australia, Perth, Western Australia, Australia \\ ${ }^{3}$ Mazankowski Alberta Heart Institute, University of Alberta, \\ Edmonton, Canada
}

Thromb Haemost 2018;118 (Suppl 1):S34-S44.

\author{
Address for correspondence C. Michael Gibson, MS, MD, Division of \\ Cardiovascular Medicine, Beth Israel Deaconess Medical Center, 20 \\ Overland Street, Suite 540, Boston, MA 02215, United States \\ (e-mail: mgibson@bidmc.harvard.edu).
}

\begin{abstract}
Keywords

- atrial fibrillation

- stroke

- oral anticoagulant

- percutaneous coronary intervention

- transcatheter aortic valve replacement

With improved life expectancy and the aging population, the global burden of atrial fibrillation (AF) continues to increase, and with AF comes an estimated fivefold increased risk of ischaemic stroke. Prophylactic anticoagulant therapy is more effective in reducing the risk of ischaemic stroke in AF patients than acetylsalicylic acid or dualantiplatelet therapy combining ASA with clopidogrel. Non-vitamin K antagonist oral anticoagulants are the standard of care for stroke prevention in patients with nonvalvular AF. The optimal anticoagulant strategy to prevent thromboembolism in AF patients who are undergoing percutaneous coronary intervention and stenting, those who have undergone successful transcatheter aortic valve replacement and those with embolic stroke of undetermined source are areas of ongoing research. This article provides an update on three randomized controlled trials of rivaroxaban, a direct, oral factor Xa inhibitor, that are complete or are ongoing, in these unmet areas of stroke prevention: oPen-label, randomized, controlled, multicentre study explorlng twO treatmeNt stratEgiEs of Rivaroxaban and a dose-adjusted oral vitamin $\mathrm{K}$ antagonist treatment strategy in patients with Atrial Fibrillation who undergo Percutaneous Coronary Intervention (PIONEER AF-PCI) trial; the New Approach riVaroxaban Inhibition of factor $X a$ in a Global trial vs Aspirin to prevenT Embolism in Embolic Stroke of Undetermined Source (NAVIGATE ESUS) trial and the Global study comparing a rivAroxaban-based antithrombotic strategy to an antipLatelet-based strategy after transcatheter aortlc vaLve rEplacement to Optimize clinical outcomes (GALILEO) trial. The data from these studies are anticipated to help address continuing challenges for a range of patients at risk of stroke.
\end{abstract}

\section{Introduction}

Stroke is the second most common cause of mortality and the third most common cause of disability worldwide. ${ }^{1}$ In 2013 , approximately 10.3 million new strokes occurred throughout the world (67\% due to ischaemic stroke), and strokes overall were shown to be associated with 113 million disability-adjusted life years (DALYs). ${ }^{2}$ Data from the Royal College of Physicians Sentinel Stroke National Audit Program (SSNAP) have shown that up to $40 \%$ of stroke survivors were left disabled and dependent on others for help with activities of daily living. ${ }^{3}$ received

September 29, 2017

accepted after revision

January 11, 2018
Copyright @ 2018 Schattauer

(1) 2018 
The global burden of stroke is also rising. Between 1990 and 2013, there was a significant increase in the number of people affected by both ischaemic and haemorrhagic stroke (an increase of 2.58 and 1.48 million cases, respectively), ${ }^{2}$ and a recent report published by the Stroke Alliance for Europe (SAFE) projects that overall there will be a $34 \%$ increase in the total number of stroke events in Europe, rising from 613,148 in 2015 to 819,771 in 2035.4

Innovative and novel strategies are required to reduce the global burden of stroke. Rivaroxaban is a non-vitamin $\mathrm{K}$ antagonist (VKA) oral anticoagulant (NOAC) that selectively and directly inhibits factor Xa, preventing thrombin formation and thereby intravascular thrombosis. ${ }^{5}$ Rivaroxaban has now shown a good safety and efficacy profile in several areas of thromboembolism, including the prevention of stroke and systemic embolism in adult patients with non-valvular atrial fibrillation (NVAF); ${ }^{6}$ the treatment of deep vein thrombosis (DVT) and pulmonary embolism (PE) and prevention of recurrent DVT and PE in adults; ${ }^{7,8}$ the prevention of venous thromboembolism (VTE) in adult patients undergoing elective hip or knee replacement surgery ${ }^{9-12}$ and the prevention of atherothrombotic events in adult patients after an acute coronary syndrome (ACS) with elevated cardiac biomarkers when co-administered with acetylsalicylic acid (ASA) alone or with ASA plus clopidogrel or ticlopidine. ${ }^{13}$

This review will cover recently completed and ongoing studies of rivaroxaban for the prevention of stroke or thrombotic events in three areas of unmet need: patients with NVAF undergoing percutaneous coronary intervention (PCI) with stenting (the oPen-label, randomized, controlled, multicentre study explorIng twO treatmeNt stratEgiEs of Rivaroxaban and a dose-adjusted oral vitamin $\mathrm{K}$ antagonist treatment strategy in patients with Atrial Fibrillation who undergo Percutaneous Coronary Intervention [PIONEER AF-PCI] trial $^{14}$ ); patients who have undergone successful transcatheter aortic valve replacement (TAVR) for aortic stenosis (AS; the Global study comparing a rivAroxaban-based antithrombotic strategy to an antipLatelet-based strategy after transcatheter aortIc vaLve rE-placement to Optimise clinical outcomes [GALILEO] trial) ${ }^{15}$ and patients with embolic stroke of undetermined source (ESUS; the New Approach riVaroxaban Inhibition of factor Xa in a Global trial vs Aspirin to prevenT Embolism in Embolic Stroke of Undetermined Source [NAVIGATE ESUS] trial). ${ }^{16}$

\section{Clinical Background: Patients with Atrial Fibrillation Undergoing Percutaneous Coronary Intervention with Stenting}

Approximately 2 to $21 \%$ of patients with ACS have underlying AF. ${ }^{17,18}$ ACS patients with AF have a two- to threefold increase in their risk of stroke, re-infarction, major bleeding and mortality when compared with those with normal sinus rhythm. ${ }^{19-21}$ Thus, the administration of an intensive combination antithrombotic strategy requires a delicate balance between the reduction of ischaemic events and an increased risk of bleeding. ${ }^{22-26}$

Current guidelines recommend administration of an oral anticoagulant (such as VKAs, apixaban, dabigatran or rivar- oxaban) in patients with NVAF for prophylaxis against ischaemic stroke. ${ }^{18,27-29}$ In the European Society of Cardiology (ESC) guidelines, a NOAC is recommended in preference to a VKA. ${ }^{28}$ Patients with ACS who undergo PCI require an alternate antithrombotic regimen. Guidelines recommend the administration of dual-antiplatelet therapy (DAPT), which consists of ASA and a $\mathrm{P}_{2} \mathrm{Y}_{12}$ inhibitor, to manage patients with ACS who undergo $\mathrm{PCI}^{30-32}$ The recommended duration of DAPT varies with the type of stent that is placed and with the patient's indication for the procedure (elective $\mathrm{PCI}$ or in the setting of an ACS), and ranges from at least 1 month for a bare metal stent to at least 6 months for a drugeluting stent, and 12 months or beyond in ACS. ${ }^{33}$ In patients with both ACS and AF, triple combination therapy is often recommended. ${ }^{18,29,34}$ This regimen, which includes an oral anticoagulant in addition to DAPT, is associated with a threeto fourfold increased risk of fatal and non-fatal bleeding. 22,24-26,35 A North American expert-based consensus on the treatment of patients with AF undergoing $\mathrm{PCI}$ recommends that the duration of DAPT, when given alongside oral anticoagulation (OAC), should not extend to a full 12 months and to consider single antiplatelet therapy starting within the first 6 months (0-6 months post stenting depending on the ischaemic/thrombotic and bleeding risk profile) for $\leq 12$ months. ${ }^{32}$ Discontinuation of antiplatelet therapy by 1 year may be considered in most patients, particularly those at low thrombotic risk or high bleeding risk. It is not known whether any single antiplatelet therapy offers additional secondary thrombotic prevention benefit in addition to OAC use beyond the early post-stent time window. The choice of OAC (VKA or NOAC; ideally administered as lifelong therapy, unless contraindicated) should be at the discretion of the treating physician, with patients informed on the risk-benefit profiles of each therapy. Given the higher risk of both ischaemic and bleeding complications early after PCI, along with OAC initiation, patients should be monitored closely during the first few months postprocedure. ${ }^{32}$

Prior to the PIONEER AF-PCI trial, there were a lack of data regarding the optimal therapy strategy in this patient population, for whom clinicians must balance the risk of stent thrombosis and ischaemic stroke with the risk of bleeding. ${ }^{14,23,36}$ The PIONEER AF-PCI trial evaluated treatment strategies similar to previous trials (in different patient populations) that demonstrated favorable benefit/risk ratios of rivaroxaban-based regimens compared with VKA-based regimens, as well as single antiplatelet regimens compared with DAPT in combination with anticoagulants. ${ }^{6,13,14,37,38}$

The What Optimal antiplatElet and anticoagulant in patients with oral anticoagulation and coronary StenTing (WOEST) trial evaluated the safety and efficacy of clopidogrel alone compared with DAPT in patients who received longterm therapy with VKA and underwent PCI. ${ }^{37}$ Patients who received clopidogrel alone demonstrated a significant reduction in bleeding compared with those on DAPT, with no increase in thrombosis. This trial suggested that, in combination with an oral anticoagulant, a single antiplatelet strategy with a $\mathrm{P}_{2} \mathrm{Y}_{12}$ inhibitor may be a safer and equally efficacious approach compared with DAPT. ${ }^{37}$ 
Several trials formed the basis of the rivaroxaban regimens studied in the PIONEER AF-PCI trial. In the Rivaroxaban Oncedaily oral direct factor Xa inhibition Compared with vitamin $\mathrm{K}$ antagonism for prevention of stroke and Embolism Trial in Atrial Fibrillation (ROCKET-AF) trial, rivaroxaban $20 \mathrm{mg}$ once daily (od) demonstrated an effective reduction in the risk of stroke in patients with NVAF, compared with VKAs. ${ }^{6}$

The Anti-Xa Therapy to Lower Cardiovascular Events in Addition to Standard Therapy in Subjects With Acute Coronary Syndrome-Thrombolysis In Myocardial Infarction 51 (ATLAS-ACS 2-TIMI 51) trial demonstrated a reduced risk of the composite endpoint of death from cardiovascular causes, myocardial infarction (MI) or stroke, and especially a reduced risk of mortality and stent thrombosis in ACS patients who received rivaroxaban $2.5 \mathrm{mg}$ twice daily (bid) in addition to single or dual-antiplatelet therapy, compared with those on antiplatelet therapy alone (ASA + either clopidogrel or ticlopidine).$^{13}$ This trial demonstrated the efficacy of rivaroxaban (2.5 mg bid) in the management of ACS. This was accompanied by an increased risk of major bleeding and intracranial haemorrhage (ICH)-but without an increased risk of fatal bleeding-compared with placebo. ${ }^{13}$

Several RCTs are ongoing that seek to elucidate the efficacy and safety of NOACs in patients with AF who undergo a PCI with stenting. ${ }^{39-41}$ The results of the WOEST, ROCKETAF and ATLAS-ACS 2-TIMI 51 trials formed the basis of the PIONEER AF-PCI trial, which addressed the unmet need of the optimal antithrombotic regimen in patients with both NVAF and coronary artery disease (CAD). ${ }^{14}$

\section{PIONEER AF-PCI Trial Design and Update}

PIONEER AF-PCI was an open-label, randomized, controlled, multicentre trial that evaluated the safety of two different regimens of rivaroxaban compared with VKA in patients with NVAF who underwent PCI with stent placement. ${ }^{14,38}$ The primary safety endpoint was the occurrence of clinically significant bleeding (Thrombolysis in Myocardial Infarction [TIMI], major or minor bleeding, or bleeding requiring medical attention [BRMA]) during the 12-month treatment period. Efficacy endpoints were secondary and included the occurrence of major adverse cardiovascular events (MACE; cardiovascular [CV] death, MI or stroke). The individual components of the primary and secondary safety endpoints and stent thrombosis were also reported. All efficacy events were adjudicated by a blinded independent clinical events committee. Exploratory endpoints included the occurrence of International Society on Thrombosis and Haemostasis (ISTH) major bleeding, Global Utilization of Streptokinase and Tissue Plasminogen Factor for Occluded Coronary Arteries (GUSTO) severe bleeding, and bleeding classified according to the Bleeding Academic Research Consortium (BARC). ${ }^{38}$

Prior to randomization, the investigators decided the intended duration of DAPT (1, 6 or 12 months) and the specific $\mathrm{P}_{2} \mathrm{Y}_{12}$ inhibitor (clopidogrel, prasugrel or ticagrelor) that would be used. A total of 2,124 patients were stratified according to the predetermined DAPT duration and randomized in a 1:1:1 ratio to one of the three treatment groups. Patients were eligible for randomization 72 hours after sheath removal and when the international normalized ratio (INR) was 2.5 or lower. Patients in Group 1 were administered a regimen consisting of rivaroxaban 15 mg od plus a P2Y $\mathrm{Y}_{12}$ inhibitor for 12 months. Patients in Group 2 received rivaroxaban $2.5 \mathrm{mg}$ bid in addition to DAPT for the prespecified duration of 1,6 or 12 months. Patients in Group 2 who received treatment for 1 or 6 months continued on rivaroxaban $15 \mathrm{mg}$ od with low-dose ASA (75-100 mg/day) for the remaining duration of the 12-month treatment period. Patients in Group 3 received a traditional 'triple therapy' regimen, which consisted of dose-adjusted VKA plus DAPT for the prespecified duration of 1,6 or 12 months. Patients in Group 3 who received treatment for 1 or 6 months received VKA with low-dose ASA for the remaining duration of the 12-month treatment period. ${ }^{38}$

In Groups 2 and 3, 15.8\% (221/1,403) of patients were assigned to 1 month of DAPT, 35.0\% $(491 / 1,403)$ to 6 months of DAPT and $49.3 \%(691 / 1,403)$ to 12 months of DAPT. The intended $\mathrm{P}_{2} \mathrm{Y}_{12}$ inhibitor for more than $90 \%$ of patients was clopidogrel. $^{38}$

At 12 months, the primary safety endpoint occurred in $16.8 \%(n=109)$ of patients in Group $1,18.0 \%(n=117)$ of patients in Group 2 and 26.7\% $(n=167)$ of patients in Group 3 (Group 1 vs. Group 3 hazard ratio [HR]: 0.59; 95\% confidence interval [CI]: 0.47-0.76; $p<0.001$ and Group 2 vs. Group 3 HR: 0.63 ; $95 \%$ CI: $0.50-0.80 ; p<0.001$; - Fig. 1). The primary safety endpoint of clinically significant bleeding occurred in 393 patients. Of those bleeding events, $85.0 \%(n=334)$ were BRMA, 6.9\% $(n=27)$ were minor bleeding events and $11.7 \%$ ( $n=46)$ were major bleeding events. Patients who received a rivaroxaban-based regimen (Groups 1 and 2) had significant reductions in BRMA compared with those who received the VKA-based regimen (Group 3: rivaroxaban vs. VKA HR: 0.64; 95\% CI: $0.51-0.80 ; p<0.001$ ). Rates of major bleeding and minor bleeding were numerically, but not significantly, lower in patients who received a rivaroxaban-based strategy compared with the VKA strategy (rivaroxaban vs. VKA major bleeding HR: $0.61 ; 95 \% \mathrm{CI}: 0.34-1.09 ; p=0.09$ and rivaroxaban vs. VKA minor bleeding HR: 0.51; 95\% CI: 0.24-1.08, $p=0.07)^{38}$

Bleeding was also assessed using ISTH, GUSTO and BARC classifications. ISTH major bleeding occurred in $3.7 \%$ $(n=52)$ of patients in Groups 1 and 2 compared with $6.9 \%$ $(n=48)$ of patients in Group $3(p=0.001)$. GUSTO severe bleeding occurred in $1.2 \%(n=17)$ of patients receiving a rivaroxaban strategy and in $2.9 \%(n=20)$ of patients who received the VKA strategy $(p=0.007)$. Furthermore, the rivaroxaban-based regimens (Groups 1 and 2) were associated with a significant reduction in the risk of BARC type $5 \mathrm{~b}$ bleeding (definitive fatal bleeding events) compared with the VKA-based regimen $(0.2$ vs. $1.0 \%, p=0.019) .{ }^{38}$

The composite MACE outcome (death from CV causes, MI or stroke) occurred in 6.5\% $(n=41)$ of patients in Group 1 , $5.6 \%(n=36)$ of patients in Group 2 and $6.0 \%(n=36)$ of patients in Group 3 (Group 1 vs. Group 3 HR: 1.08; 95\% CI: $0.69-1.68 ; p=0.75$ and Group 2 vs. Group 3 HR: 0.93; $95 \%$ CI: $0.59-1.48 ; p=0.76)$. Rates of the individual components 


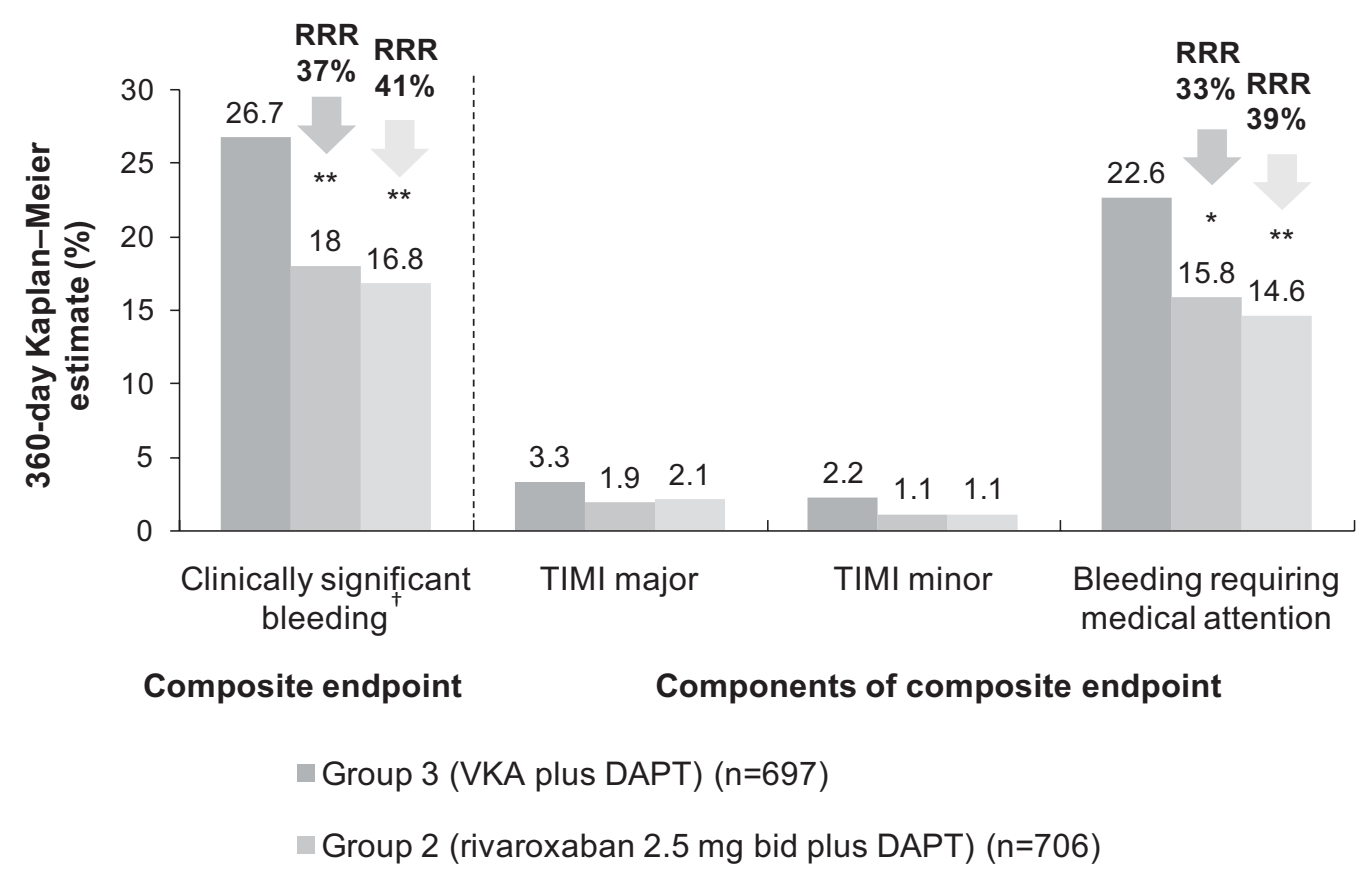

Fig. 1 Key safety outcomes in the PIONEER AF-PCI trial. ${ }^{38}$ bid, twice daily; DAPT, dual-antiplatelet therapy; RRR, relative risk reduction; TIMI, thrombolysis in myocardial infarction; VKA, vitamin $\mathrm{K}$ antagonist. ${ }^{*} p=0.002$ vs. Group $3 ;{ }^{* *} p<0.001$ vs. Group 3 ; ${ }^{\dagger}$ composite of TIMI major bleeding, TIMI minor bleeding and bleeding requiring medical attention.

of secondary efficacy endpoint and stent thrombosis were similar across all groups $(p>0.05) .^{38}$

\section{Re-hospitalization Results}

In a retrospective analysis of the PIONEER AF-PCI data, the occurrence of all-cause death or re-hospitalization for an adverse event (AE) was assessed as a primary endpoint. ${ }^{42}$ The risk of occurrence of the primary endpoint was $34.9 \%$ $(n=228)$ in Group 1, 31.9\% $(n=213)$ in Group 2 and 41.9\% $(n=261)$ in Group 3 (Group 1 vs. Group 3 HR: 0.79; 95\% CI: $0.66-0.94 ; p=0.008$ and Group 2 vs. Group 3 HR: 0.75 ; 95\% CI: $0.62-0.90 ; p=0.002$ ). Additionally, the rate of all-cause re-hospitalization was $34.1 \%$ in Group $1,31.2 \%$ in Group 2 and 41.5\% in Group 3 (Group 1 vs. Group 3 HR: 0.77; 95\% CI: $0.65-0.92 ; p=0.005$ and Group 2 vs. Group 3 HR: $0.74 ; 95 \% \mathrm{CI}: 0.61-0.88 ; p=0.001) .^{42}$

Further reductions were observed in the risk of all-cause death and bleeding as a cause of re-hospitalization (Group 1 vs. Group 3 HR: 0.69; 95\% CI: 0.48-0.97; $p=0.032$ and Group 2 vs. Group 3 HR: $0.64 ; 95 \%$ CI: $0.45-0.91 ; p=0.012$ ) and in the risk of all-cause death and re-hospitalization for cardiovascular reasons (Group 1 vs. Group 3 HR: 0.70; 95\% CI: $0.56-0.87 ; p=0.001$ and Group 2 vs. Group 3 HR: $0.75 ; 95 \%$ CI: $0.60-0.94 ; p=0.011) .^{42}$

Based on the results of PIONEER AF-PCI, the European Medicines Agency (EMA) approved the use of rivaroxaban $15 \mathrm{mg}$ od (or $10 \mathrm{mg}$ od for patients with moderate renal impairment [creatinine clearance $30-49 \mathrm{~mL} / \mathrm{min}$ ]) in combination with a $\mathrm{P}_{2} \mathrm{Y}_{12}$ inhibitor for a maximum of 12 months' duration for the treatment of patients with NVAF who require oral anticoagulation and undergo $\mathrm{PCI}$ with stent placement. $^{5}$

\section{Clinical Background: Embolic Stroke of Undetermined Source}

Cryptogenic stroke is a category of ischaemic stroke for which no cause is found, ${ }^{43}$ partly due to incomplete diagnostic assessment of the stroke, or where more than one possible cause is identified. Epidemiological studies have reported that cryptogenic stroke accounts for around 25\% of cases of ischaemic stroke, and is found in a younger age group than those with non-cryptogenic stroke. ${ }^{43}$ Advances in imaging techniques and improved understanding of stroke pathophysiology have prompted a reassessment of cryptogenic stroke. There is persuasive evidence that most cryptogenic strokes are thromboembolic. ${ }^{43}$ In 2014 , this led to the term embolic stroke of undetermined source, or ESUS, which was defined by the Cryptogenic Stroke/ESUS International Working Group as a non-lacunar brain infarct without proximal arterial stenosis or a major cardioembolic source. ${ }^{44}$ ESUS refers to patients with embolic stroke for whom the etiology of embolism remains unknown, despite standard (not advanced or specialized) investigations to determine the source. ${ }^{43-45}$ The prevalence of ESUS is not consistent in the published literature due to variations in the definition and degree of investigation, but overall, about one in six ischaemic strokes are ESUS. ${ }^{44,46}$

The potential origin of thromboembolism underlying ESUS includes several well-established embolic sources, such as minor-risk or covert cardiac sources, veins via paradoxical embolism, and nonocclusive atherosclerotic plaques in the aortic arch, cervical or cerebral arteries. ${ }^{44}$ A standardized evaluation is required to exclude possible causes of embolic stroke to place a patient in the clinical category of 
ESUS. ${ }^{43-45}$ This requirement has led to the development of clinical diagnostic algorithms for ESUS. ${ }^{44}$

A diagnosis of ESUS is made as follows: stroke detected by computed tomography (CT) or magnetic resonance imaging (MRI) that is not lacunar (lacunar defined as subcortical infarction $\leq 1.5 \mathrm{~cm}$ on $\mathrm{CT}$ or $\leq 2.0 \mathrm{~cm}$ on diffusion-weighted [DW] MRI and in the distribution of a small penetrating cerebral artery); absence of vascular imaging evidence of extracranial or intracranial arterial atherosclerosis causing more than 50\% luminal stenosis in arteries supplying the ischaemic area; no electrocardiographic and echocardiographic evidence of major-risk cardioembolic source of embolism (e.g., permanent or paroxysmal AF, sustained atrial flutter, intracardiac thrombus, prosthetic cardiac valve, atrial myxoma or other cardiac tumours, mitral stenosis, MI within the past 4 weeks, left ventricular ejection fraction [LVEF] $<30 \%$, valvular vegetations or infective endocarditis); and no clinical, imaging and laboratory evidence of other specific causes of stroke, such as arteritis, dissection, migraine, vasospasm or drug misuse. ${ }^{44}$

There is an increased risk of recurrent stroke in patients with ESUS, which has been estimated at a rate of 3 to $6 \%$ per year (estimated at 1-2\% per year in younger [average age: mid-40s] patients taking ASA and as high as $14 \%$ per year in older patients). ${ }^{44}$ In the Athens Stroke Registry, stroke recurrence in patients with ESUS was reported to be $29.0 \%$ (95\% CI: 22-36\%) over 5 years, which was similar to the risk of recurrence of cardioembolic stroke (26\%) and significantly higher when compared with all other non-cardioembolic subtypes of stroke. ${ }^{47}$

Currently, there are no clinical guidelines specifically for the management of ESUS. The 2012 American College of Chest Physicians (ACCP) guidelines on ischaemic stroke, the 2014 AHA/American Stroke Association [ASA] guidelines for the management of TIA and stroke, and the 2008 European Stroke Organization (ESO) guidelines are currently extrapolated to the management of ESUS and amongst them recommend antiplatelet therapy, lifestyle modification and control of other potential risk factors, such as hypertension, diabetes and dyslipidaemia for patients with non-cardioembolic stroke. ${ }^{48-50}$

Because the annual rate of recurrent stroke after ESUS is considerable despite current best medical therapy (usually ASA) ${ }^{44}$ there is a clear need for more effective stroke prevention in patients with ESUS.

\section{Navigate ESUS}

NAVIGATE ESUS was a Phase III, double-blind, randomized, controlled trial, established to assess the efficacy and safety of rivaroxaban versus ASA for the secondary prevention of stroke and systemic embolism in patients with recent ESUS. ${ }^{16,51}$

Patients were eligible for inclusion if they were 50 years or older and had been diagnosed with ESUS in the previous 7 days to 6 months. ${ }^{16,51}$ In this trial, ESUS was diagnosed as a non-lacunar sub-cortical ischaemic stroke or TIA confirmed by neuroimaging, with the absence of stenotic intracranial or extracranial carotid artery atherosclerosis greater than $50 \%$ or occlusion; exclusion of a diagnosis of $\mathrm{AF}$ after 20-hour cardiac monitoring and no detectable intracardiac thrombus seen on imaging with trans-oesophageal or transthoracic echocardiography, and no other detectable cause of ischaemic stroke after standard investigation. Patients were excluded if they had an indication for chronic anticoagulation or antiplatelet therapy. ${ }^{16,51}$

Patients were randomized in a 1:1 ratio to rivaroxaban $15 \mathrm{mg}$ od or ASA $100 \mathrm{mg}$ od after the qualifying ESUS. ${ }^{16,51}$ The primary efficacy measure was the composite of the first occurrence of all recurrent strokes (including TIA with positive neuroimaging) and systemic embolic events. The primary safety measure was the first occurrence of a major bleeding event according to the criteria of the ISTH. The trial design is described in further detail in - Fig. 2. Between December 2014 and September 2017, a total of 7,214 patients were enrolled from 459 centres across 31 countries. ${ }^{52}$ Mean patient follow-up was expected to be about 2 years, with the trial continuing until at least 450 participants had experienced a primary efficacy outcome event-initially expected to be February 2018. ${ }^{16,51}$ However, in October 2017, the decision was taken by the Academic Leadership of the trial and the sponsor, Bayer AG, for the trial to be stopped early, based on a recommendation by the Independent Data Monitoring Committee following a planned interim analysis. ${ }^{52}$ Results showed comparable efficacy between the rivaroxaban and ASA arms, with minimal chance of rivaroxaban showing overall benefit if the study was fully completed. While bleeding rates were low overall, an increase in bleeding was observed in the rivaroxaban arm compared with the low-dose ASA arm. Therefore, the hypothesis that anticoagulation is better than antiplatelet therapy in patients with ESUS could not be confirmed by the NAVIGATE ESUS trial. A complete data analysis is expected in 2018. ${ }^{52}$ Other ongoing RCTs investigating NOACs in similar patients include the following: Randomized, double-blind, Evaluation in secondary Stroke Prevention comparing the EfficaCy and safety of the oral Thrombin inhibitor dabigatran etexilate versus acetylsalicylic acid in patients with Embolic Stroke of Undetermined Source (RE-SPECT ESUS) ${ }^{53,54}$ and Apixaban for Treatment of Embolic Stroke of Undetermined Source (ATTICUS). ${ }^{55,56}$

\section{Clinical Background: Transcatheter Aortic Valve Replacement}

Degenerative AS is a condition that increases with age and is now the most common form of valvular heart disease (VHD) in developed countries. ${ }^{57-59}$ In elderly individuals 75 years or older, the prevalence of AS is estimated to be as high as $12.4 \%$ in Europe and North America. ${ }^{58}$ A study undertaken in Norway revealed that AS is a progressive disease accelerating both with age and degree, with a mean annual increase of $3.2 \mathrm{~mm} \mathrm{Hg}$ in mean transvalvular pressure gradient. ${ }^{57}$ Aortic valve replacement (AVR) is the only effective treatment option for symptomatic AS. ${ }^{57}$ Surgical aortic valve replacement (SAVR) procedures require the use of sternotomy and cardiopulmonary bypass, whereas TAVR (also known as transcatheter aortic valve intervention) is less invasive using the trans-femoral, trans-apical or trans-subclavian approach or alternative vascular approaches. ${ }^{60,61}$ TAVR is currently established as an alternative therapy in prohibitive risk, 


\section{NAVIGATE ESUS}

Objective: Efficacy of rivaroxaban in secondary prevention of stroke and prevention of systemic embolism in patients with a recent ESUS

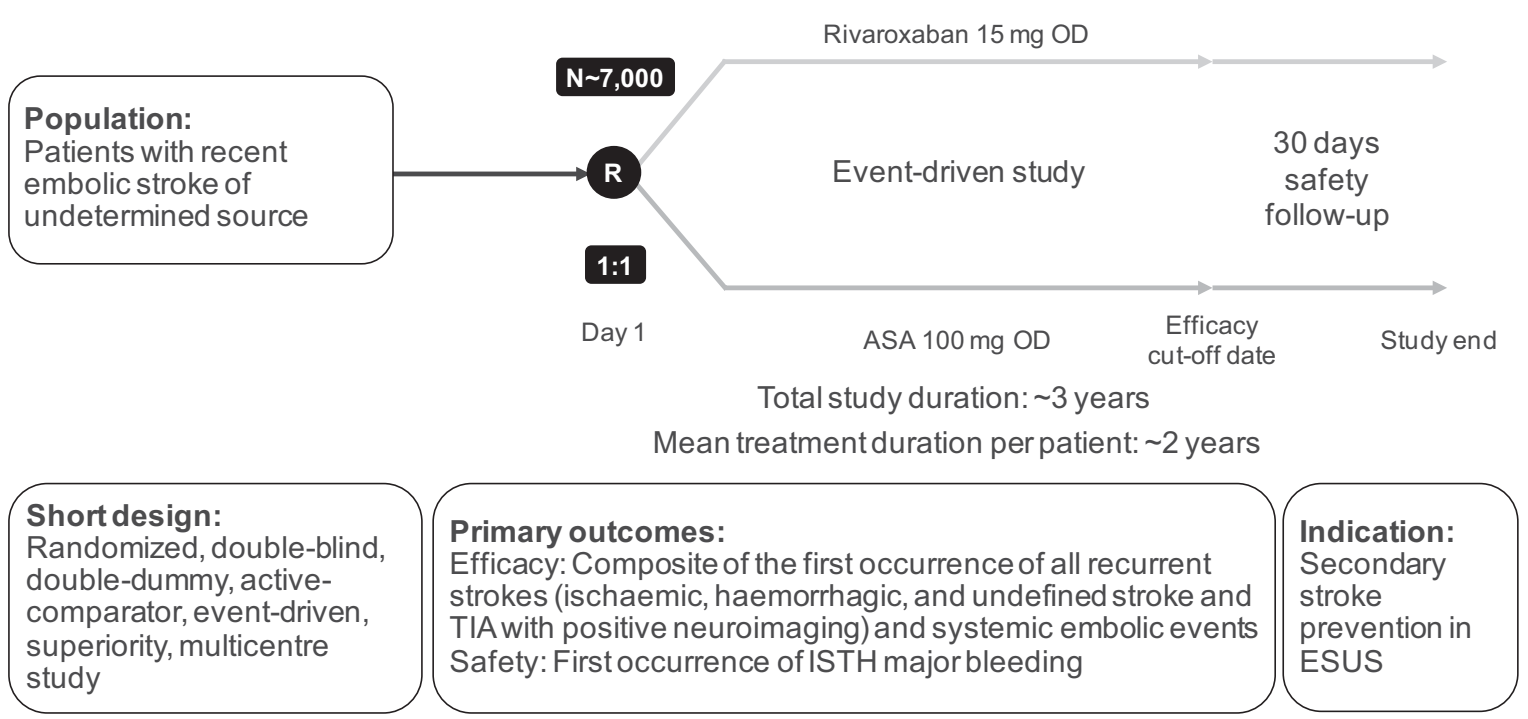

Fig. 2 NAVIGATE ESUS trial design. ${ }^{16,51}$ ASA, acetylsalicylic acid; ESUS, embolic stroke of undetermined source; ISTH, International Society on Thrombosis and Haemostasis; od, once daily; R, randomized; TIA, transient ischaemic attack.

high-risk and moderate-risk patients ${ }^{62,63}$ with ongoing investigation in low-risk populations. ${ }^{64}$

Data have emphasized the risks patients may face following TAVR, namely stroke or transient ischaemic attack (TIA) within 30 days, which has been reported at a rate of $3.3 \pm 1.8 \%$, with the majority being major strokes $(2.9 \pm 1.8 \%) .{ }^{60}$ Furthermore, patients undergoing TAVR may be at risk of late bleeding events. ${ }^{60}$

There are now data from clinical trials and real-world studies, which suggest that clinical outcomes following TAVR are somewhat similar to or even better than SAVR. In 2016, the results from the Placement of Aortic Transcatheter Valves 2A (PARTNER-2A) trial were published, which was a randomized trial of 2,032 intermediate-risk patients from the United States and Canada who underwent TAVR or SAVR. ${ }^{62}$ The incidence of all-cause mortality or disabling stroke was similar between the two groups at all time points assessed ( 30 days, 1 year and 2 years; $p=0.001$ for non-inferiority overall). Consistently similar rates were also noted between TAVR and SAVR groups for both stroke (30 days: 5.5 vs. $6.1 \%, p=0.57 ; 1$ year: 8.0 vs. $8.1 \%, p=0.88 ; 2$ years: 9.5 vs. $8.9 \%, p=0.67$, respectively) and disabling stroke ( 30 days: 3.2 vs. $4.3 \%, p=0.20 ; 1$ year: 5.0 vs. $5.8 \%, p=0.46 ; 2$ years: 6.2 vs. $6.4 \%, p=0.83$, respectively). ${ }^{62}$ These findings were supported by real-world data from an observational study of patient outcomes following TAVR with the use of the SAPIEN 3 valve. ${ }^{65} \mathrm{~A}$ total of 1,077 patients with severe AS who were at intermediate risk of surgical mortality undergoing TAVR or SAVR were investigated. TAVR was both non-inferior $(p<0.0001)$ and superior $(p<0.0001)$ to SAVR for the primary composite endpoint of mortality, stroke and moderate-to-severe aortic regurgitation at 1 year following a propensity score analysis, indicating that TAVR could have benefits over SAVR in this patient group. ${ }^{65}$

In the PARTNER-2A trial, TAVR was associated with lower incidences of new-onset AF (NOAF), acute kidney injury and life-threatening or disabling bleeding at 30 days compared with SAVR; however, major vascular complications at 30 days were significantly higher following TAVR versus SAVR at 30 days (7.9 vs. $5.0 \%, p=0.008){ }^{62}$ The earlier PARTNER trial, undertaken in high-risk patients with severe AS undergoing TAVR or SAVR, also showed an increase in major vascular complications at 30 days with TAVR over SAVR, which was statistically significant (11.0vs. $3.2 \%, p<0.001) .{ }^{63}$ While the rates of major stroke were similar between high-risk groups at 30 days (3.8 vs. $2.1 \%$, respectively; $p=0.20$ ), TAVR was shown to be associated with a significant increase in major stroke at 1 year compared with SAVR (5.1 vs. $2.4 \%$, respectively; $p=0.07$ ). ${ }^{63}$ Clearly, with the increasing use of TAVR comes the increasing challenge of balancing thromboembolic and bleeding complications, depending on the patients' clinical risk profile.

Another consequence following TAVR is that thromboemboli may originate directly from the valve structure and/or leaflets, ${ }^{66}$ which can arise owing to the valve platform used to support the transcatheter valve implantation. While symptomatic bioprosthetic aortic valve thrombosis is reported to have a prevalence of 1 to $2 \%$, subclinical valve leaflet thrombosis following biosynthetic AVR occurs more frequently, with a prevalence of 10 to $15 \%{ }^{67}$ Subclinical leaflet thrombosis following TAVR and SAVR is defined as the presence of reduced leaflet motion, with hypo-attenuated leaflet lesions seen on 
imaging. ${ }^{67}$ Current management guidelines do not give recommendations for antithrombotic treatment for subclinical leaflet thrombosis, due to the limited evidence from clinical studies. $^{68-70}$

A recent study investigated the effects of NOAC therapy on 931 patients with subclinical leaflet thrombosis who underwent TAVR or SAVR and included patient data from two registries: Assessment of Transcatheter and Surgical Aortic Bioprosthetic Valve Thrombosis and its Treatment with Anticoagulation (RESOLVE; 657 patients; 71\%) and Subclinical Aortic Valve Bioprosthesis Thrombosis Assessed with Four-Dimensional Computed Tomography (SAVORY; 274 patients; 29\%). ${ }^{67}$ Subclinical leaflet thrombosis was lower in patients receiving anticoagulants (4\% [8/224]) compared with those receiving DAPT (15\% [31/208]; $p<0.0001)$ and NOACs were equally as effective as warfarin (3\% [3/107] vs. $4 \%$ [5/117]; $p=0.72)$. Subclinical leaflet thrombosis resolved in $100 \%$ (36/36) of patients when anticoagulants were initiated (warfarin: $n=24$; NOACs: $n=12$ ), but persisted in $91 \%(20 / 22)$ of patients not receiving anticoagulants $(p<0.0001)$. Although stroke rates were not different between those with or without reduced leaflet motion, subclinical valve leaflet thrombosis was associated with increased rates of both TIA (4.18 vs. 0.60 TIAs per 100 person-years; $p=0.0005$ ) and all strokes or TIAs ( 7.85 vs. 2.36 per 100 person-years; $p=0.001$ ). ${ }^{67}$ These findings highlight both the importance of recognizing subclinical leaflet thrombosis following TAVR and the potential, but as yet undefined role for NOACs in the prevention of post-TAVR thromboembolism, TIA and stroke.

For patients who are undergoing TAVR, and who do not require anticoagulation, current clinical guidelines from the ESC and the European Association for Cardio-Thoracic Surgery (EACTS), and the American College of Cardiologists (ACC)/ American Heart Association (AHA), recommend DAPT for 3 to 6 months following TAVR, followed by indefinite ASA therapy. ${ }^{68-70}$ However, these guidelines are based on expert consensus rather than clinical trials. The extensive WoRldwIde TAVI ExperieNce (WRITTEN) survey, from 250 centres in 38 countries, with a cumulative experience of nearly 70,000 TAVRs, showed that post-TAVR antithrombotic treatment regimens were highly variable. ${ }^{71}$ Additionally, these recommendations are not supported by recent data-the Aspirin versus Aspirin + Clopidogrel Following Transcatheter Aortic Valve Implantation (ARTE) trial showed that single-antiplatelet therapy with ASA alone may prove more beneficial than DAPT following TAVR in terms of reducing the occurrence of major $A E s$, reducing the risk for major or life-threatening events and not contributing to an increase in the risk for MI or stroke. ${ }^{72}$

Currently, there are no clinical guidelines recommending any specific antithrombotic regimen for stroke prevention following TAVR. ${ }^{68-70}$ The use of TAVR is increasing, and it is anticipated that the procedure will replace SAVR as the standard of care in the majority of patients over the next decade. It is unknown whether an antiplatelet or anticoagulant strategy is more appropriate for the prevention of thromboembolic events following TAVR. ${ }^{73}$ There are several ongoing trials that are investigating different antithrombotic strategies after TAVR-namely Anti-Thrombotic Strategy
After Trans-Aortic Valve Implantation for Aortic Stenosis (ATLANTIS) and Edoxaban Compared with Standard Care After Heart Valve Replacement Using a Catheter in Patients With Atrial Fibrillation (ENVISAGE TAVI). ${ }^{73-75}$ In the Randomized, Phase II Study to Evaluate the Safety and Pharmacokinetics of Oral Dabigatran Etexilate in Patients after Heart Valve Replacement (RE-ALIGN) trial, dabigatran, when used in patients with mechanical heart valves, was associated with increased rates of thromboembolic and bleeding complications versus warfarin. ${ }^{76}$ The GALILEO trial will evaluate whether a rivaroxaban-based anticoagulation strategy, compared with an antiplatelet-based strategy, is more effective in reducing death or first thromboembolic events for patients who have undergone successful TAVR. ${ }^{15}$

\section{GALILEO Trial Design}

GALILEO is a Phase III, multicentre, open-label, international, randomized, event-driven trial that includes approximately 1,520 patients who have undergone successful TAVR. ${ }^{15,77}$ The rationale for GALILEO is to determine the optimal antithrombotic treatment following TAVR and to determine whether rivaroxaban may reduce the risk of thromboembolic complications post-TAVR with an acceptable risk of bleeding compared with an antiplatelet-based strategy in subjects without need of chronic oral anticoagulation. ${ }^{15,77}$

The trial design includes randomization in a $1: 1$ ratio, between 1 and 7 days following a successful TAVR, to either a rivaroxaban-based treatment strategy or an antiplateletbased strategy. ${ }^{15,77}$ In the experimental arm, patients will receive rivaroxaban $10 \mathrm{mg}$ od plus ASA 75 to $100 \mathrm{mg}$ od for 90 days, followed by rivaroxaban $10 \mathrm{mg}$ alone. In the control arm, patients will receive ASA 75 to $100 \mathrm{mg}$ od plus clopidogrel $75 \mathrm{mg}$ od for 90 days, followed by ASA alone. ${ }^{15,77}$ The GALILEO trial design is described in further detail in - Fig. 3 .

Because of the possibility that patients in the trial may develop NOAF following randomization, the following adaptations will be made. ${ }^{15,77}$ If NOAF develops, the rivaroxaban dose will be raised from 10 to $20 \mathrm{mg}$ od, or from 10 to $15 \mathrm{mg}$ od for subjects with moderate renal impairment (estimated glomerular filtration rate [GFR] $<50$ and $\geq 30 \mathrm{~mL} / \mathrm{min}$ per $1.73 \mathrm{~m}^{2}$ ). If NOAF occurs within the first 90 days in subjects randomized to the rivaroxaban strategy, ASA will be discontinued at 90 days, and rivaroxaban will be continued as monotherapy. If NOAF occurs under the clopidogrel-based strategy, clopidogrel ( $\leq 90$ days) or ASA monotherapy ( $>90$ days) will be replaced by a VKA to target an INR of 2 to 3. If NOAF occurs within the first 90 days in this group, ASA will be discontinued at 90 days, and VKA continued as a monotherapy.

The primary efficacy endpoint of GALILEO will be the composite of stroke, MI, symptomatic valve thrombosis, PE, DVT, non-central nervous system (CNS) systemic embolism and all-cause death. ${ }^{15,77}$ The primary safety endpoint will be the composite of disabling, life-threatening and major bleeding events, according to Valve Academic Research Consortium-2 (VARC-2) definitions. ${ }^{78}$ Recruitment began in December 2015 , from approximately 144 centres across 15 countries, with an anticipated completion date of $2018 .^{15,77}$ 


\section{GALILEO}

Objective: To assess a rivaroxaban-based anticoagulation regimen following successful TAVR balancing ischemic and bleeding outcome measures

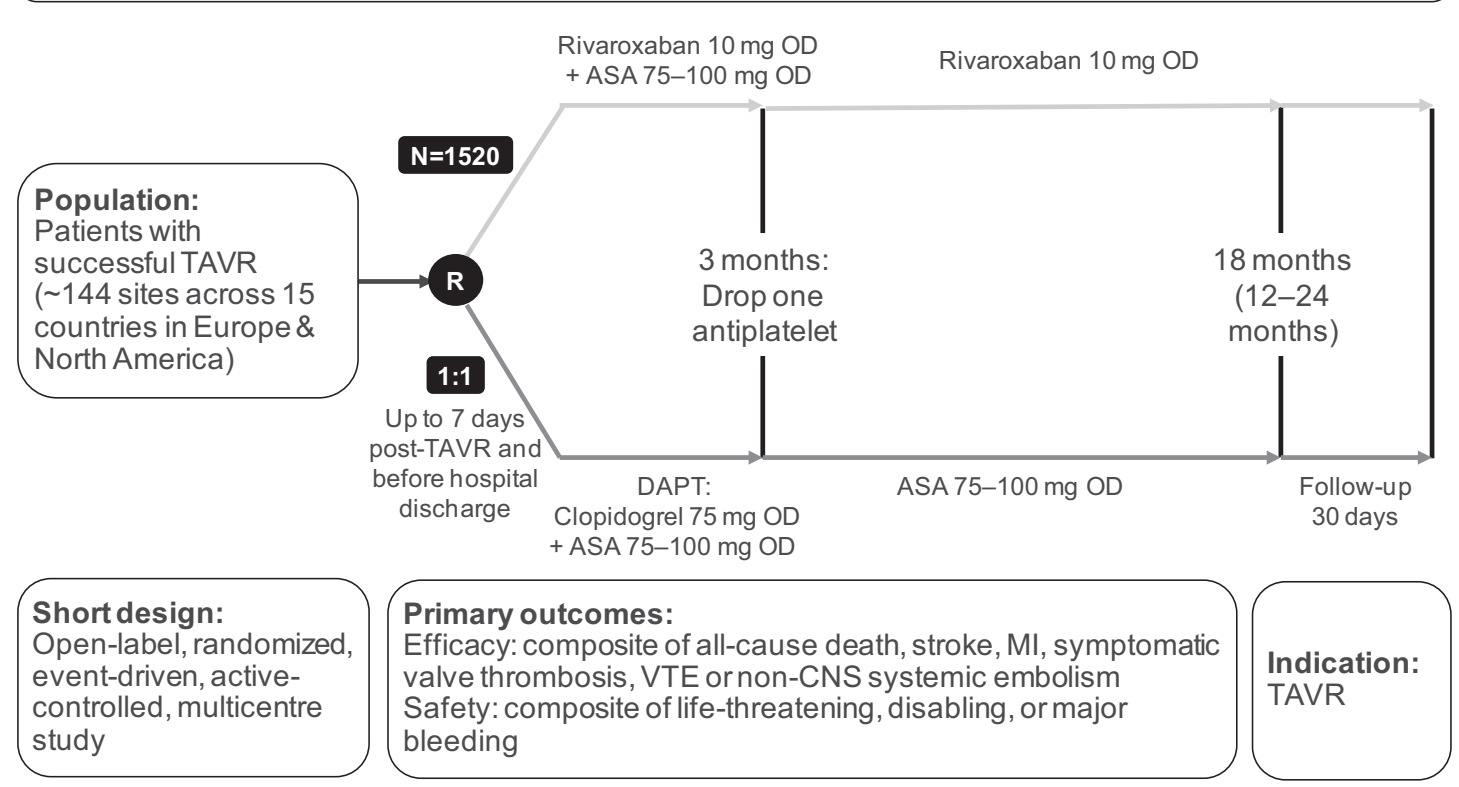

Fig. 3 GALILEO trial design. ${ }^{15,77}$ ASA, acetylsalicylic acid; CNS, central nervous system; DAPT, dual-antiplatelet therapy; MI, myocardial infarction; od, once daily; R, randomized; TAVR, transcatheter aortic valve replacement; VTE, venous thromboembolism.

\section{Conclusion}

It is clear that there are a wide range of patients at risk of stroke as a result of undergoing various procedures or owing to the presence of specific risk factors. For these patients, data are scarce in terms of informing the most appropriate management approach. Data from the landmark PIONEER AF-PCI trial provide initial clarity around using NOACs to treat patients with $\mathrm{AF}$ who are undergoing $\mathrm{PCI}$ with stenting, leading to the approval of rivaroxaban $15 \mathrm{mg}$ od plus a $\mathrm{P}_{2} \mathrm{Y}_{12}$ inhibitor, particularly owing to the safety benefits observed. It is hoped that other ongoing trials of rivaroxaban will improve on the currently limited knowledge on optimal antithrombotic treatment after TAVR and in patients with ESUS. These trials are likely to present clinicians with a more comprehensive picture around the role of NOACs such as rivaroxaban, especially with regards to their safety profile when used in patients for whom thrombotic risk continues to be a challenge.

\section{Funding}

Editorial support was funded by Bayer AG.

\section{Conflicts of Interest}

All authors confirm that they have full access to data and contributed to drafting of the paper. C.M.G. has received research grant support from Johnson \& Johnson Corporation, Bayer AG and Portola Pharmaceuticals, and has acted in the capacity of consultant (with moderate support) from
Johnson \& Johnson Corporation, Bayer AG and Portola Pharmaceuticals. G.J.H. has received honoraria from Bayer AG for lecturing at sponsored scientific symposia and serving on advisory boards about stroke prevention in atrial fibrillation. G.J.H. was also a member of the Executive Committee of the ROCKET AF trial.

T.N. has no disclosures to report. R.C.W. is a consultant for Bayer AG, Pfizer/Bristol-Myers Squibb and Amgen Canada; has received research grants from AstraZeneca, Bayer AG and Boehringer-Ingelheim; and has received honoraria from AstraZeneca and Bayer AG.

\section{Acknowledgements}

The authors would like to acknowledge Kelly Farrell at Ketchum (Inspired Science), who provided editorial support with funding from Bayer AG.

\section{References}

1 Feigin VL, Forouzanfar MH, Krishnamurthi R, et al; Global Burden of Diseases, Injuries, and Risk Factors Study 2010 (GBD 2010) and the GBD Stroke Experts Group. Global and regional burden of stroke during 1990-2010: findings from the Global Burden of Disease Study 2010. Lancet 2014;383(9913):245-254

2 Feigin VL, Krishnamurthi RV, Parmar P, et al; GBD 2013 Writing Group; GBD 2013 Stroke Panel Experts Group. Update on the Global Burden of Ischemic and Hemorrhagic Stroke in 19902013: The GBD 2013 Study. Neuroepidemiology 2015;45(03): $161-176$ 
3 Royal College of Physicians, Clinical Effectiveness and Evaluation Unit on behalf of the Intercollegiate Stroke Working Party. Sentinel Stroke National Audit Programme (SSNAP): Clinical Audit August-November 2016 Public Report; 2017

4 King's College London for the Stroke Alliance for Europe. The burden of stroke in Europe. Available at: https://www.stroke.org. uk/sites/default/files/theburdenofstrokeineuropereport.pdf. Accessed February 8, 2018

5 Bayer AG. Xarelto Summary of Product Characteristics. Available at: http://www.ema.europa.eu/docs/en_GB/document_library/ EPAR_-_Product_Information/human/000944/WC500057108.pdf. Accessed February 8, 2018

6 Patel MR, Mahaffey KW, Garg J, et al; ROCKET AF Investigators. Rivaroxaban versus warfarin in nonvalvular atrial fibrillation. N Engl J Med 2011;365(10):883-891

7 Bauersachs R, Berkowitz SD, Brenner B, et al; EINSTEIN Investigators. Oral rivaroxaban for symptomatic venous thromboembolism. N Engl J Med 2010;363(26):2499-2510

8 Büller HR, Prins MH, Lensin AW, et al; EINSTEIN-PE Investigators. Oral rivaroxaban for the treatment of symptomatic pulmonary embolism. N Engl J Med 2012;366(14):1287-1297

9 Eriksson BI, Borris LC, Friedman RJ, et al; RECORD1 Study Group. Rivaroxaban versus enoxaparin for thromboprophylaxis after hip arthroplasty. N Engl J Med 2008;358(26):2765-2775

10 Kakkar AK, Brenner B, Dahl OE, et al; RECORD2 Investigators. Extended duration rivaroxaban versus short-term enoxaparin for the prevention of venous thromboembolism after total hip arthroplasty: a double-blind, randomised controlled trial. Lancet 2008;372(9632):31-39

11 Lassen MR, Ageno W, Borris LC, et al; RECORD3 Investigators. Rivaroxaban versus enoxaparin for thromboprophylaxis after total knee arthroplasty. N Engl J Med 2008;358(26):2776-2786

12 Turpie AG, Lassen MR, Davidson BL, et al; RECORD4 Investigators. Rivaroxaban versus enoxaparin for thromboprophylaxis after total knee arthroplasty (RECORD4): a randomised trial. Lancet 2009;373(9676):1673-1680

13 Mega JL, Braunwald E, Wiviott SD, et al; ATLAS ACS 2-TIMI 51 Investigators. Rivaroxaban in patients with a recent acute coronary syndrome. N Engl J Med 2012;366(01):9-19

14 Gibson CM, Mehran R, Bode C, et al. An open-label, randomized, controlled, multicenter study exploring two treatment strategies of rivaroxaban and a dose-adjusted oral vitamin $\mathrm{K}$ antagonist treatment strategy in subjects with atrial fibrillation who undergo percutaneous coronary intervention (PIONEER AF-PCI). Am Heart J 2015;169(04):472-8.e5

15 Windecker S, Tijssen J, Giustino G, et al. Trial design: rivaroxaban for the prevention of major cardiovascular events after transcatheter aortic valve replacement: rationale and design of the GALILEO study. Am Heart J 2017;184:81-87

16 Hart RG, Sharma M, Mundl H, et al. Rivaroxaban for secondary stroke prevention in patients with embolic strokes of undetermined source: design of the NAVIGATE ESUS randomized trial. European Stroke Journal 2016;1(03):146-154

17 Schmitt J, Duray G, Gersh BJ, Hohnloser SH. Atrial fibrillation in acute myocardial infarction: a systematic review of the incidence, clinical features and prognostic implications. Eur Heart J 2009;30 (09):1038-1045

18 January CT, Wann LS, Alpert JS, et al; American College of Cardiology/American Heart Association Task Force on Practice Guidelines. 2014 AHA/ACC/HRS guideline for the management of patients with atrial fibrillation: a report of the American College of Cardiology/ American Heart Association Task Force on Practice Guidelines and the Heart Rhythm Society. J Am Coll Cardiol 2014;64(21):e1-e76

19 Crenshaw BS, Ward SR, Granger CB, Stebbins AL, Topol EJ, Califf RM. Atrial fibrillation in the setting of acute myocardial infarction: the GUSTO-I experience. Global Utilization of Streptokinase and TPA for Occluded Coronary Arteries. J Am Coll Cardiol 1997; 30(02):406-413
20 Hersi A, Alhabib KF, Alsheikh-Ali AA, et al. Prognostic significance of prevalent and incident atrial fibrillation among patients hospitalized with acute coronary syndrome: findings from the Gulf RACE-2 Registry. Angiology 2012;63(06):466-471

21 Mehta RH, Dabbous OH, Granger CB, et al; GRACE Investigators. Comparison of outcomes of patients with acute coronary syndromes with and without atrial fibrillation. Am J Cardiol 2003;92 (09):1031-1036

22 Faxon DP, Eikelboom JW, Berger PB, et al. Consensus document: antithrombotic therapy in patients with atrial fibrillation undergoing coronary stenting. A North-American perspective. Thromb Haemost 2011;106(04):572-584

23 Hemmrich M, Peterson ED, Thomitzek K, Weitz JI. Spotlight on unmet needs in stroke prevention: The PIONEER AF-PCI, NAVIGATE ESUS and GALILEO trials. Thromb Haemost 2016;116 (Suppl 2):S33-S40

24 Huber K, Airaksinen KJ, Cuisset T, Marín F, Rubboli A, Lip GY. Antithrombotic therapy in patients with atrial fibrillation undergoing coronary stenting: similarities and dissimilarities between North America and Europe. Thromb Haemost 2011;106(04):569-571

25 Lamberts M, Gislason GH, Olesen JB, et al. Oral anticoagulation and antiplatelets in atrial fibrillation patients after myocardial infarction and coronary intervention. J Am Coll Cardiol 2013;62 (11):981-989

26 Lip GY, Huber K, Andreotti F, et al; European Society of Cardiology Working Group on Thrombosis. Management of antithrombotic therapy in atrial fibrillation patients presenting with acute coronary syndrome and/or undergoing percutaneous coronary intervention/stenting. Thromb Haemost 2010;103(01):13-28

27 Camm AJ, Lip GY, De Caterina R, et al; ESC Committee for Practice Guidelines (CPG). 2012 focused update of the ESC Guidelines for the management of atrial fibrillation: an update of the 2010 ESC Guidelines for the management of atrial fibrillation. Developed with the special contribution of the European Heart Rhythm Association. Eur Heart J 2012;33(21):2719-2747

28 Macle L, Cairns J, Leblanc K, et al; CCS Atrial Fibrillation Guidelines Committee. 2016 Focused Update of the Canadian Cardiovascular Society Guidelines for the Management of Atrial Fibrillation. Can J Cardiol 2016;32(10):1170-1185

29 You JJ, Singer DE, Howard PA, et al. Antithrombotic therapy for atrial fibrillation: Antithrombotic Therapy and Prevention of Thrombosis, 9th ed: American College of Chest Physicians Evidence-Based Clinical Practice Guidelines. Chest 2012;141(2, Suppl):e531S-e575S

30 Windecker S, Kolh P, Alfonso F, et al; Authors/Task Force members. 2014 ESC/EACTS Guidelines on myocardial revascularization: The Task Force on Myocardial Revascularization of the European Society of Cardiology (ESC) and the European Association for Cardio-Thoracic Surgery (EACTS)Developed with the special contribution of the European Association of Percutaneous Cardiovascular Interventions (EAPCI). Eur Heart J 2014;35 (37):2541-2619

31 Levine GN, Bates ER, Blankenship JC, et al. 2011 ACCF/AHA/SCAI Guideline for Percutaneous Coronary Intervention: a report of the American College of Cardiology Foundation/American Heart Association Task Force on Practice Guidelines and the Society for Cardiovascular Angiography and Interventions. Circulation 2011;124(23):e574-e651

32 Angiolillo DJ, Goodman SG, Bhatt DL, et al. Antithrombotic therapy in patients with atrial fibrillation undergoing percutaneous coronary intervention: a North American Perspective2016 update. Circ Cardiovasc Interv 2016;9(11):e004395

33 Levine GN, Bates ER, Bittl JA, et al. 2016 ACC/AHA Guideline Focused Update on Duration of Dual Antiplatelet Therapy in Patients With Coronary Artery Disease: A Report of the American College of Cardiology/American Heart Association Task Force on Clinical Practice Guidelines. J Am Coll Cardiol 2016;68(10): 1082-1115 
34 Camm AJ, Kirchhof P, Lip GY, et al; European Heart Rhythm Association; European Association for Cardio-Thoracic Surgery. Guidelines for the management of atrial fibrillation: the Task Force for the Management of Atrial Fibrillation of the European Society of Cardiology (ESC). Eur Heart J 2010;31(19): 2369-2429

35 Hansen ML, Sørensen R, Clausen MT, et al. Risk of bleeding with single, dual, or triple therapy with warfarin, aspirin, and clopidogrel in patients with atrial fibrillation. Arch Intern Med 2010;170 (16):1433-1441

36 Capodanno D, Angiolillo DJ. Management of antiplatelet and anticoagulant therapy in patients with atrial fibrillation in the setting of acute coronary syndromes or percutaneous coronary interventions. Circ Cardiovasc Interv 2014;7(01):113-124

37 Dewilde WJ, Oirbans T, Verheugt FW, et al; WOEST study investigators. Use of clopidogrel with or without aspirin in patients taking oral anticoagulant therapy and undergoing percutaneous coronary intervention: an open-label, randomised, controlled trial. Lancet 2013;381(9872):1107-1115

38 Gibson CM, Mehran R, Bode C, et al. Prevention of bleeding in patients with atrial fibrillation undergoing PCI. N Engl J Med 2016;375(25):2423-2434

39 REDUAL-PCI. ClinicalTrials.gov Identifier: NCT02164864. Available at: https://ClinicalTrials.gov/show/NCT02164864. Accessed February 8, 2018

40 AUGUSTUS. ClinicalTrials.gov Identifier: NCT02415400. Available at: https://clinicaltrials.gov/ct2/show/NCT02415400. Accessed February 8, 2018

41 ENTRUST AF-PCI. ClinicalTrials.gov Identifier: NCT02866175. Available at: https://clinicaltrials.gov/ct2/show/NCT02866175. Accessed February 8, 2018

42 Gibson CM, Pinto DS, Chi G, et al. Recurrent hospitalization among patients with atrial fibrillation undergoing intracoronary stenting treated with 2 treatment strategies of rivaroxaban or a doseadjusted oral vitamin K antagonist treatment strategy. Circulation 2017;135(04):323-333

43 Saver JL. Clinical practice. Cryptogenic stroke. N Engl J Med 2016; 374(21):2065-2074

44 Hart RG, Diener HC, Coutts SB, et al; Cryptogenic Stroke/ESUS International Working Group. Embolic strokes of undetermined source: the case for a new clinical construct. Lancet Neurol 2014; 13(04):429-438

45 Hart RG, Catanese L, Perera KS, Ntaios G, Connolly SJ. Embolic stroke of undetermined source: a systematic review and clinical update. Stroke 2017;48(04):867-872

46 Perera KS, Vanassche T, Bosch J, et al; ESUS Global Registry Investigators. Embolic strokes of undetermined source: prevalence and patient features in the ESUS Global Registry. Int J Stroke 2016;11(05):526-533

47 Ntaios G, Papavasileiou V, Milionis H, et al. Embolic strokes of undetermined source in the Athens Stroke Registry: an outcome analysis. Stroke 2015;46(08):2087-2093

48 Lansberg MG, O'Donnell MJ, Khatri P, et al. Antithrombotic and thrombolytic therapy for ischemic stroke: Antithrombotic Therapy and Prevention of Thrombosis, 9th ed: American College of Chest Physicians Evidence-Based Clinical Practice Guidelines. Chest 2012;141(2, Suppl):e601S-e636S

49 Kernan WN, Ovbiagele B, Black HR, et al; American Heart Association Stroke Council, Council on Cardiovascular and Stroke Nursing, Council on Clinical Cardiology, and Council on Peripheral Vascular Disease. Guidelines for the prevention of stroke in patients with stroke and transient ischemic attack: a guideline for healthcare professionals from the American Heart Association/American Stroke Association. Stroke 2014;45(07):2160-2236

50 European Stroke Organisation (ESO) Executive Committee; ESO Writing Committee. Guidelines for management of ischaemic stroke and transient ischaemic attack 2008. Cerebrovasc Dis 2008;25(05):457-507
51 NAVIGATE-ESUS. ClinicalTrials.gov Identifier: NCT02313909. Available at: https://clinicaltrials.gov/ct2/show/ NCT02313909. Accessed February 8, 2018

52 Bayer AG. Bayer's NAVIGATE ESUS study halted early as it indicated comparable efficacy between treatment arms. Available at: http://press.bayer.com/baynews/baynews.nsf/id/C45F4AFB2CEFBDD0C12581B0004CF01B/\$File/2017-0301E.pdf?open\&$\bmod =06.10 .2017 \_08: 49: 26$. Accessed February 8, 2018

53 RE-SPECT ESUS. ClinicalTrials.gov Identifier: NCT02239120. Available at: https://clinicaltrials.gov/ct2/show/NCT02239120. Accessed February 8, 2018

54 Diener HC, Easton JD, Granger CB, et al; RE-SPECT ESUS Investigators. Design of Randomized, double-blind, Evaluation in secondary Stroke Prevention comparing the EfficaCy and safety of the oral Thrombin inhibitor dabigatran etexilate vs. acetylsalicylic acid in patients with Embolic Stroke of Undetermined Source (RE-SPECT ESUS). Int J Stroke 2015;10(08):1309-1312

55 ATTICUS. ClinicalTrials.gov Identifier: NCT02427126. Available at: https://clinicaltrials.gov/ct2/show/NCT02427126. Accessed February 8, 2018

56 Geisler T, Poli S, Meisner C, et al. Apixaban for treatment of embolic stroke of undetermined source (ATTICUS randomized trial): rationale and study design. Int J Stroke 2017;12(09): 985-990

57 Eveborn GW, Schirmer H, Heggelund G, Lunde P, Rasmussen K. The evolving epidemiology of valvular aortic stenosis: the Tromsø study. Heart 2013;99(06):396-400

58 Osnabrugge RL, Mylotte D, Head SJ, et al. Aortic stenosis in the elderly: disease prevalence and number of candidates for transcatheter aortic valve replacement: a meta-analysis and modeling study. J Am Coll Cardiol 2013;62(11):1002-1012

59 Eggebrecht $\mathrm{H}$, Mehta RH. Transcatheter aortic valve implantation (TAVI) in Germany 2008-2014: on its way to standard therapy for aortic valve stenosis in the elderly? EuroIntervention 2016;11 (09):1029-1033

60 Eggebrecht H, Schmermund A, Voigtländer T, Kahlert P, Erbel R, Mehta RH. Risk of stroke after transcatheter aortic valve implantation (TAVI): a meta-analysis of 10,037 published patients. EuroIntervention 2012;8(01):129-138

61 Ye J, Soon JL, Webb J. Aortic valve replacement vs. transcatheter aortic valve implantation: patient selection. Ann Cardiothorac Surg 2012;1(02):194-199

62 Leon MB, Smith CR, Mack MJ, et al; PARTNER 2 Investigators. Transcatheter or surgical aortic-valve replacement in intermediate-risk patients. N Engl J Med 2016;374(17):1609-1620

63 Smith CR, Leon MB, Mack MJ, et al; PARTNER Trial Investigators. Transcatheter versus surgical aortic-valve replacement in highrisk patients. N Engl J Med 2011;364(23):2187-2198

64 PARTNER-3. ClinicalTrials.gov identifier: NCT02675114. Available at: https://clinicaltrials.gov/ct2/show/NCT02675114. Accessed February 8, 2018

65 Thourani VH, Kodali S, Makkar RR, et al. Transcatheter aortic valve replacement versus surgical valve replacement in intermediaterisk patients: a propensity score analysis. Lancet 2016;387 (10034):2218-2225

66 Makkar RR, Fontana G, Jilaihawi H, et al. Possible subclinical leaflet thrombosis in bioprosthetic aortic valves. N Engl J Med 2015;373(21):2015-2024

67 Chakravarty T, Søndergaard L, Friedman J, et al; RESOLVE; SAVORY Investigators. Subclinical leaflet thrombosis in surgical and transcatheter bioprosthetic aortic valves: an observational study. Lancet 2017;389(10087):2383-2392

68 Vahanian A, Alfieri O, Andreotti F, et al; Joint Task Force on the Management of Valvular Heart Disease of the European Society of Cardiology (ESC); European Association for CardioThoracic Surgery (EACTS). Guidelines on the management of valvular heart disease (version 2012). Eur Heart J 2012;33(19): 2451-2496 
69 Nishimura RA, Otto CM, Bonow RO, et al; ACC/AHA Task Force Members. 2014 AHA/ACC Guideline for the Management of Patients With Valvular Heart Disease: executive summary: a report of the American College of Cardiology/American Heart Association Task Force on Practice Guidelines. Circulation 2014; 129(23):2440-2492

70 Nishimura RA, Otto CM, Bonow RO, et al. 2017 AHA/ACC Focused Update of the 2014 AHA/ACC Guideline for the Management of Patients With Valvular Heart Disease: A Report of the American College of Cardiology/American Heart Association Task Force on Clinical Practice Guidelines. Circulation 2017;135(25):e1159-e1195

71 Cerrato E, Nombela-Franco L, Nazif TM, et al. Evaluation of current practices in transcatheter aortic valve implantation: The WRITTEN (WoRldwIde TAVIExperieNce) survey. Int J Cardiol 2017;228:640-647

72 Rodés-Cabau J, Masson JB, Welsh RC, et al. Aspirin versus aspirin plus clopidogrel as antithrombotic treatment following transcatheter aortic valve replacement with a balloon-expandable valve: The ARTE (Aspirin Versus Aspirin + Clopidogrel Following Transcatheter Aortic Valve Implantation) Randomized Clinical Trial. JACC Cardiovasc Interv 2017;10(13):1357-1365
73 Capodanno D, Angiolillo DJ. Antithrombotic therapy for prevention of cerebral thromboembolic events after transcatheter aortic valve replacement: evolving paradigms and ongoing directions. JACC Cardiovasc Interv 2017;10(13):1366-1369

74 ATLANTIS. ClinicalTrials.gov Identifier: NCT02664649. Available at: https://clinicaltrials.gov/ct2/show/NCT02664649. Accessed February 8, 2018

75 ENVISAGE-TAVI AF. ClinicalTrials.gov Identifier: NCT02943785. Available at: https://clinicaltrials.gov/ct2/show/NCT02943785. Accessed February 8, 2018

76 Eikelboom JW, Connolly SJ, Brueckmann M, et al; RE-ALIGN Investigators. Dabigatran versus warfarin in patients with mechanical heart valves. N Engl J Med 2013;369(13):1206-1214

77 GALILEO. ClinicalTrials.gov Identifier: NCT02556203. Available at: https://clinicaltrials.gov/ct2/show/NCT02556203. Accessed February 8, 2018

78 Kappetein AP, Head SJ, Généreux P, et al. Updated standardized endpoint definitions for transcatheter aortic valve implantation: the Valve Academic Research Consortium-2 consensus document. J Am Coll Cardiol 2012;60(15):1438-1454 